\title{
Radiological Study of Portal Vein Variations and its Morphometry with Special Reference to its Surgical Implications of Liver
}

\author{
Vidya C S, Manjunatha B, ${ }^{2}$ Sudha Kiran Das ${ }^{3}$ \\ 1Professor Department of Anatomy JSS Medical College, JSS AHER, Mysuru-15 \\ ${ }^{2}$ Professor of Forensic Medicine, JSS AHER, Mysuru-15 \\ ${ }^{3}$ Professor of Radiology JSS AHER, Mysuru-15
}

Disclose and conflicts of interest: none to be declared by all authors

\begin{abstract}
Introduction: the blood supply to liver is supplied by hepatic artery which contributes to about $25 \%$ to $30 \%$. and from the portal vein which contributes to the remaining $70 \%-75 \%$. The variations in portal venous anatomy and dimensions of portal veins are important in liver related procedures like transplant, resections, Trans jugular Intra parenchymal Porto-Systemic Shunt (TIPSS) and radiological intervention procedures.

Objective: to describe the anatomic variations in branching pattern of portal vein by CECT scan method. To study the morphometry of portal vein and its major branches (length, diameter) by 3D reconstruction of CECT scan method.

Method: 80 CECT scan images of patients was collected from the Radiology Department to evaluate variations in branching pattern of portal vein. Axial and post processed 2D and 3D reformations contributed for accurate evaluation of morphometry of major portal veins.

Results: type I pattern of portal vein was observed in $87.5 \%$, Type II in $10 \%$ of cases and Type III in $2.5 \%$ of cases. There was no gender difference in branching pattern and for variations of portal vein. The mean \pm SD of length of main portal vein, right portal vein and left portal vein were $3.8 \pm 0.94,1.69 \pm 0.4,2.91 \pm 0.71$ respectively and diameters of main portal vein, right portal vein and left portal vein were $0.98 \pm 0.13,0.68 \pm 0.08,0.50 \pm 0.04$ respectively.

Conclusion: this study highlights the branching pattern and dimensions of portal veins which is prerequisite for hepatobliliary and liver transplantation surgeries.
\end{abstract}

Keywords: Portal veins; Liver; CECT scan; Liver surgeries.

\section{Introduction}

The liver being the largest organ in human body accounts to $2 \%$ to $3 \%$ of average body weight. Liver is located in the right upper quadrant of the abdominal cavity which is well protected by the thoracic cage and maintains its position through peritoneal reflections and various ligaments. ${ }^{1}$ Morphologically and functionally liver has been divided into two lobes; right and left lobes demarcated by falciform ligament. But on basis of external appearance liver is divided into four lobes which are: right, left, quadrate, and caudate lobes. ${ }^{2}$

The liver is a highly vascular organ which has dual blood supply, $25 \%$ to $30 \%$ supplied by the hepatic artery and 70 to $75 \%$ supplied by portal vein. ${ }^{3}$ The portal vein is approximately $8 \mathrm{~cm}$ long and ascends obliquely to the right behind the first part of the duodenum and enters the porta hepatis dividing into right and left main branches within the lesser omentum.

The right branch of the portal vein is only $2-3 \mathrm{~cm}$ in length and usually divides into anterior division supplies segments V and VIII, and a right posterior division supplies segments VI and VII. The left portal vein has a longer extrahepatic course $(4-5 \mathrm{~cm})$ than the right portal vein, tends to lie more horizontal, and is often smaller in calibre. It has a horizontal portion that runs along the inferior surface of segment IV and gives branches to segment I and sometimes to segment IV. The left branch of the portal vein continues within the liver, giving off branches to II and III. ${ }^{4}$

Because of complex vascular anatomy of liver there is high incidence of variants and in $10-15 \%$ of livers right branch of portal vein variation is common. ${ }^{5}$ The variations of portal veins is quite important in segmental liver resections of liver which is a curative treatment for hepatocellular carcinoma. ${ }^{6}$ According to Nakamura classification of portal veins Type A-The usual bifurcation of main portal vein into right and left portal vein, Type B- Trifurcation of main portal vein into right anterior, right posterior and left portal vein, Type C- Right posterior branch arising proximally from main portal vein and main portal vein bifurcating right posterior branch and left portal vein, Type DRight posterior branch arising separately from the left portal vein Type E- Branches of segment V and VIII originating separately from the left portal vein. ${ }^{7}$ 
Embryologically, the portal vein is formed by selective involution of the vitelline veins, which have two anastomoses anterior and posterior anastomosis. The proximal ventral anastomoses forms left branch of portal vein, the dorsal anastomoses forms main portal vein. The distal anastomoses usually disappears. Any alteration in pattern of these anastomoses will result in portal vein variations. ${ }^{8}$

Portal hypertension is caused due to Budd Chiari syndrome, cirrhosis of liver, veno-occlusive disease, and congenital hepatic fibrosis. The life saving procedure to prevent esophageal variceal bleeding is transjugular intraportosystemic shunt(TIPS). To decide the size of stent, accurate dimensions of portal veins and hepatic veins is important for successful portocaval shunting procedure. ${ }^{9}$ Presence of variations in portal venous anatomy should never be a surprise to the surgeon and detailed imaging studies need to be performed on the patient before planning the surgery. Hence the present study aims to determine portal vein variations and dimensions by Contrast enhanced CT scan method which will help the hepatobiliary surgeons to plan, modify as needed and execute a complex surgery in a safe manner.

\section{Material and Method}

Cross sectional study was conducted in 80 patients at JSS Medical College, Mysore. Ethical clearance was obtained from Institution ethical Committee and informed consent was obtained from patients for CECT images. Contrast Enhanced CT was performed on Philips Ingenuity Core 128 (Philips Healthcare Co, Ltd, PMS, Cleveland Inc, US). Scans were obtained in venous phase with the threshold set at 120HU. Images of about $3 \mathrm{~mm}$ thickness were observed and additional images were reconstructed with $0.625 \mathrm{~mm}$ reconstruction intervals for detailed interpretation. Definitions of portal vein and its tributaries were observed by skilled Radiologists and variations of the same were documented. Axial source images remained the basis for observing portal venous anatomy, however post processed 2D and 3D reformations contributed for accurate evaluation. The morphometric measurements of vessels (length, diameter) were analyzed in sagittal and coronal planes of multiplanar processed images.

Inclusion criteria: All the abdominal contrast enhanced CT scan images without liver lacerations were included for the study.

Exclusion criteria: Those patients with extrahepatic malignancy, cholangiocarcinoma or diffuse portal vein (PV) thrombosis were excluded from the study

Statistical Analysis: Frequency of variations of portal veins was calculated and expressed as percentages. The descriptive Statistics (Mean, SD) were obtained for length and diameter of Portal veins. Analyzed data was expressed as Mean \pm SD. The differences between means were interpreted statistically significant at $\mathrm{p}<0.05$. Mann Whitney $U$ test was performed to see the significant difference between variants (Type I vs Type II) for length and diameter of Portal Veins. The whole statistical analysis was performed using SPSS version 22 (licensed to JSS AHER).

\section{Results}

The normal anatomy the portal vein (Type I) was observed in $87.5 \%$ by CECT scan method while the remaining showed variant branching pattern.(Figure 1) The most common variation observed was Type II, where there was trifurcation of portal vein into right anterior portal vein, right posterior portal vein and left portal vein. This variation was seen in $10 \%$ (Figure 2) and 2.5\% cases for Type III (separate origin of right posterior portal vein from main portal vein, then common trunk which divided into right anterior portal vein and left portal vein) as shown in Figure 3. There was no gender difference in branching pattern and for variations of portal vein.

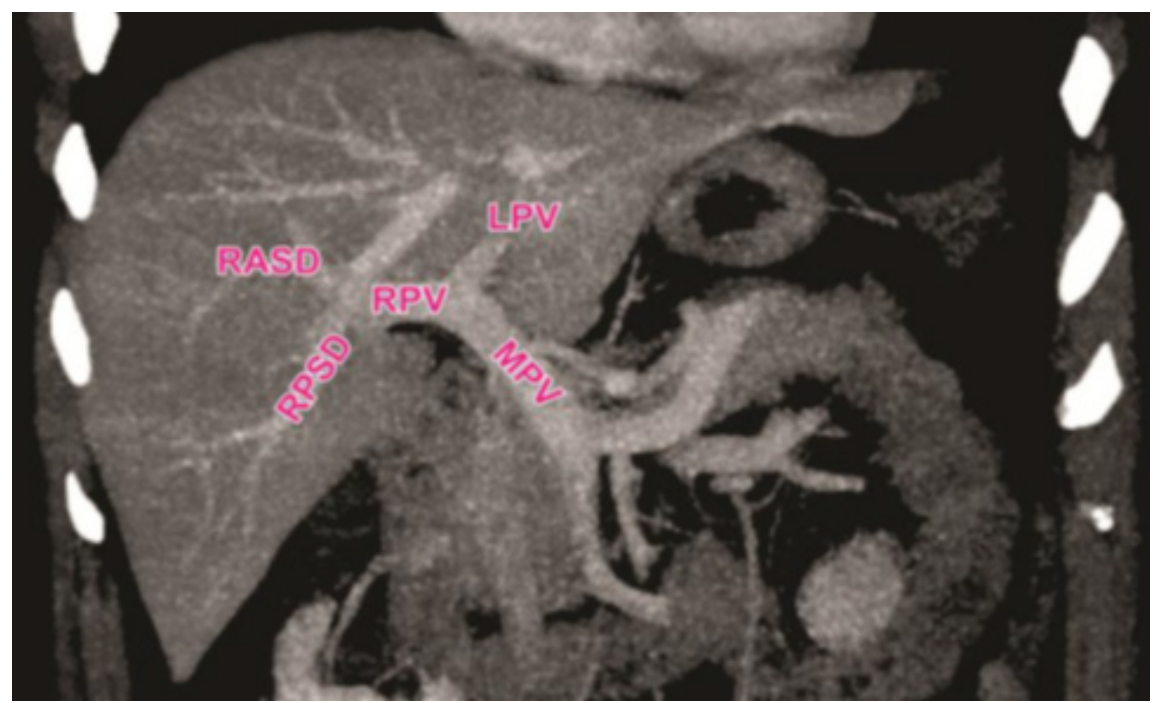

Figure 1. Showing Coronal CECT image with Type I portal vein-Main portal vein(MPV) divides into right and left portal veins. The right portal vein RPV divides into right anterior and right posterior veins. The left portal vein (LPV) divides into medial and lateral branches 




Figure 2. Showing Type II or trifurcation of portal veins into right anterior, right posterior and LPV by cast and axial image of CECT scan

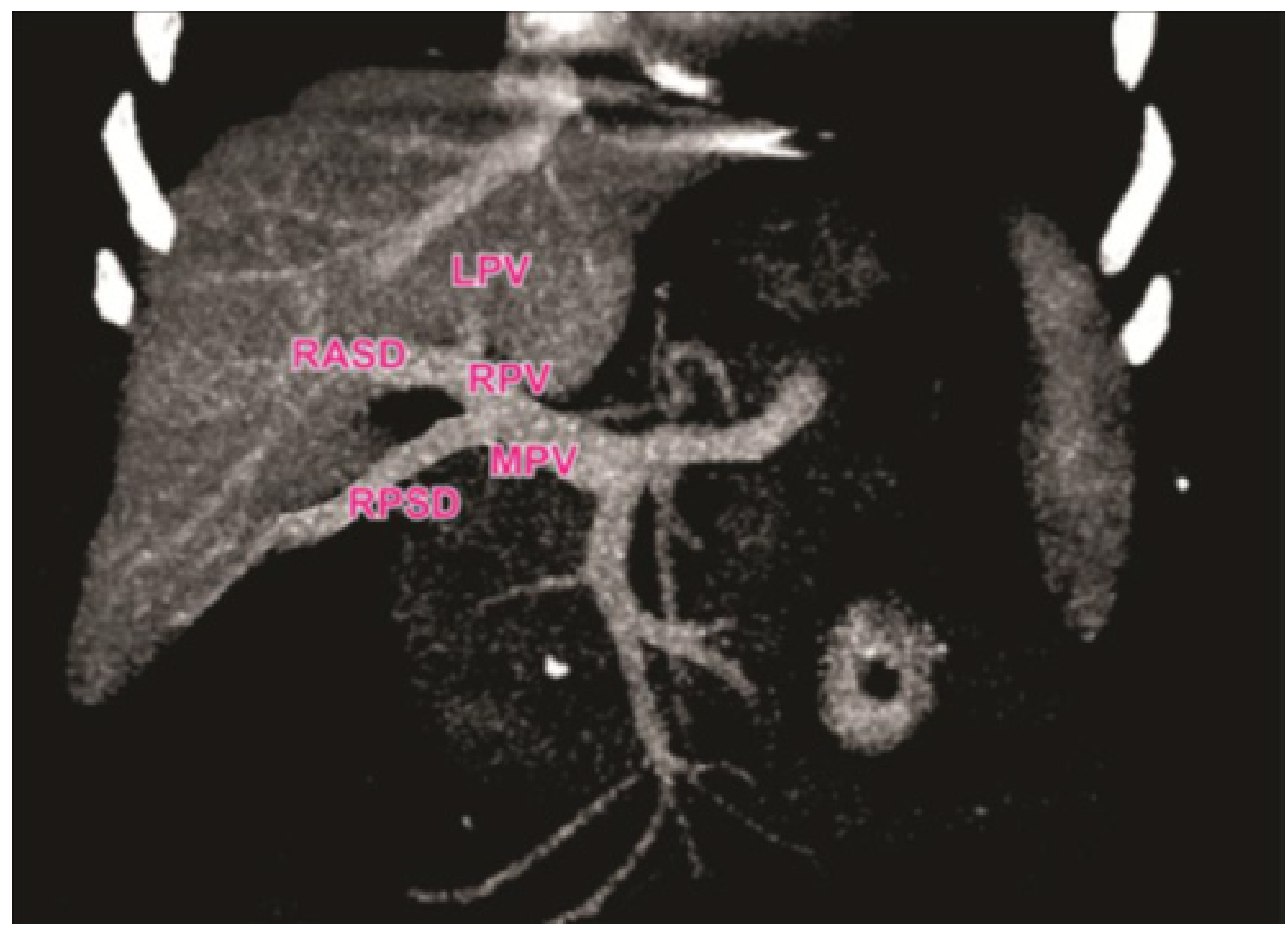

Figure 3. Showing Type III or right posterior vein arising from MPV in oblique coronal image of CECT scan

The length and diameters of MPV, RPV and LPV were analyzed using descriptive statistics as shown in Table 1. The length and diameters of portal veins (MPV, LPV, RPV) were compared for Type I and
Type II variants. There was no significant difference ( $>0.05$ ) observed in length and diameters in Type II variants compared to Type I as shown in Table 2. 
Table 1. Showing Mean \pm SD for length and diameters of portal veins by CECT scan method $(n=80)$

\begin{tabular}{|c|c|c|c|}
\hline \multirow{2}{*}{ Parameters } & \multirow{2}{*}{ Portal veins } & \multicolumn{2}{|c|}{ CECT Method } \\
\hline & & Min-Max & Mean \pm SD \\
\hline \multirow{3}{*}{ Length (in cms) } & Main Portal Vein & $2.1-5.9$ & $3.80 \pm 0.94$ \\
\hline & Left Portal Vein & $2.1-4.3$ & $2.91 \pm 0.71$ \\
\hline & Right Portal Vein & $0.9-3.1$ & $1.69 \pm 0.40$ \\
\hline \multirow{3}{*}{ Diameter (in cms) } & Main Portal Vein & $0.7-1.2$ & $0.98 \pm 0.13$ \\
\hline & Left Portal Vein & $0.4-0.6$ & $0.5 \pm 0.04$ \\
\hline & Right Portal Vein & $0.5-0.83$ & $0.68 \pm 0.08$ \\
\hline
\end{tabular}

Table 2. Showing comparison of length and diameters of portal veins in Type I vs Type II variants

\begin{tabular}{|c|c|c|c|c|c|}
\hline \multirow{2}{*}{ Parameter } & \multirow{2}{*}{ Methods } & Type I & Type II & \multirow{2}{*}{$\mid \mathbf{| Z |}$} & \multirow{2}{*}{ p-value } \\
\hline & & Mean Rank & Mean Rank & & \\
\hline \multirow{3}{*}{ Length (in cms) } & Main portal vein & 40.70 & 29.70 & 1.040 & 0.299 \\
\hline & Left Portal Vein & 40.35 & 34.80 & 0.527 & 0.598 \\
\hline & Right Portal Vein & 41.01 & 25.10 & 1.507 & 0.132 \\
\hline \multirow{3}{*}{ Diameter(in cms) } & Main portal vein & 40.96 & 25.80 & 1.449 & 0.147 \\
\hline & Left Portal Vein & 39.86 & 42.00 & 0.203 & 0.839 \\
\hline & Right Portal Vein & 38.76 & 58.30 & 1.848 & 0.065 \\
\hline
\end{tabular}

\section{Discussion}

Liver transplantation involves implant of a whole or part of a donor liver into arecipient with liver failure. Classically a liver transplant operation involves three vascular anastomosis and One biliary anastomosis. The vascular anastomosis includes- two inflows namely Hepatic artery Portal vein and one venous outflow. Division of the liver in the donor is wholly dependent on the Vascular anatomy as the parenchymal transection follows vascular planes. Any variation in the vascular anatomy will alter surgical plans, both in the donor and in the recipient during implantation for example, trifurcation of portal vein (Type II) needs double anastomosis. Rarely portal vein anomalies in the donor might even contra-indicate donation where the blood supply to the remnant donor liver may be compromised. ${ }^{10}$ Example include, in trifurcation of portal vein right hepatectomy is contraindicated. The incidence of portal vein variations in the liver and the surgical implications are increasingly obvious. ${ }^{11}$ With the growing popularity of complex hepatobiliary surgical and percutaneous portal vein embolization procedures, the detection of portal vein variants are increasingly relevant. In LDLT, careful handling of the vascular system is critical to avoid causing injury to portal vein in the residual liver. ${ }^{12,13,14}$

In the present study the classical Type I portal vein was the most commonly encountered and was observed in $87.5 \%$, Type II in $10 \%$ and Type III in $2.5 \%$. In previous studies by Kishi Type I was observed in 91\% and Type II 9\% of cases. ${ }^{15}$ Takeishi and others reported Type I in 89\%, Type II in 6.1\% and Type III in $4.7 \% .{ }^{16}$ Hence our present study findings for Type I is in concordance to Takeishi. Type I variant observed by Sureka was $80 \%$, Type II in $6.83 \%$, Type III in $4.9 \%$ , Type IV in $2.69 \%$ and Type V in $1 \% .{ }^{17}$ Convey and others reported Type I in 65\%, Type II in 9\%, Type III in $13 \%$ and Type $\mathrm{V}$ in $6 \%$. Hence our present study the prevalence of variations of Type II was in accordance to study conducted by Convey. ${ }^{18}$

In the present study, length of Main portal vein was 2.1-5.9 cms, length of the Right Portal Vein ranged from 0.9 to $3.1 \mathrm{cms}$ and length of the Left portal vein ranged from $2.1-4.3 \mathrm{cms}$. The diameter main portal vein was $0.7-1.2 \mathrm{~cm}$, right portal vein $0.5-0.8$ and left portal vein 0.4-0.6 respectively. Abdoulaye Kante et al studied morphometry of main portal veins in 1000 cases by Ultrasound and reported that in $29.8 \%$ of the cases, the length of the main portal vein was between 61 and $70 \mathrm{~mm}$ and in $8.8 \%$ between 81 and $100 \mathrm{~mm}$. The transverse diameter of the portal vein in its origin varied between 8 and $10 \mathrm{~mm}$ in $57.9 \%$ of the cases. The transverse diameter of the portal vein near its termination varied between 8 and $10 \mathrm{~mm}$ in $56.9 \%$ cases. $^{19}$

Harsimarjith Kaur reported the average length of main portal was found to be $62.9 \mathrm{~mm}$ and average breadth of Portal vein at its midpoint between its formation and termination was found to be $16.8 \mathrm{~mm} .^{20}$ In previous study reported by Arora the length of Right portal vein was $1.3-2.3 \mathrm{cms}^{21}$

The variations of portal veins is important in procedures such as liver transplants for appropriate vascular ligation and anastomosis. Portal hypertension 
is caused due to Budd Chiari syndrome, cirrhosis of liver, veno-occlusive disease, and congenital hepatic fibrosis. Transjugular Intrahepatic Portosystemic Shunt is a procedure that uses imaging guidance to connect the portal vein to the hepatic vein in the liver. ${ }^{9}$ Hence the morphometry of portal veins is essential to prevent potential complications during shunting procedure.

\section{Conclusion}

The present anatomical data helps surgical gastroenterologists and laparoscopic surgeons to modify surgical approach in order to achieve satisfactory outcome. Preoperative imaging in detection of vascular variants in both donor and recipient serves as a major role in planning the liver transplantation. Hence detailed knowledge of course, diameter, branches and possible variants of portal veins is a prerequisite for surgeons and radiologists. This study offers morphometric data of portal veins which can be used to compare the data with other groups globally.

\section{Limitations}

The main limitation of Contrast enhanced CT scan to visualize 3 dimension portal venous anatomy of liver is absence of appropriate software.

\section{References}

1. Sherif RZ, Abdel-Misihand Mark Bloomston. Liver Anatomy Surg $\mathrm{Cl}$ in North Am. 2010;90(4):643-653.

2. Skandalakis JE et al. Hepatic surgical anatomy. SurgClin North Am. 2004;84(2):413-435.

3. Blumgart LH, Belghiti J. Surgery of the liver, biliary tract, and pancreas.3rd edition.Philadelphia: Saunders Elsevier.2007;3-30.

4. Sabiston text book of surgery. The biological basis of modern surgical practice.Elsevier publications. First South Asia edition. 2008;1411-1467. 5. Susan Standring.Gray's Anatomy. The Anatomical Basis of clinical practice, 40thedition. 2008;11073-74.

6. Guido Torzilliet al. Total or partial anatomical resection of segment 8 using the ultrasound guided finger compression technique. HPB.2011; Vol 13: 586-591.

7. Nakamura T et al: Anatom-ical variations and surgical strategies in right lobe living donor liver. Transplantation 2002;73:1896-1903.

8. Langman's. Medical Embryology 13th edition.T.W.Sadler.Wolters Kulwer.2015;205.

9. Michael J Zinnori, Stanley W Ashley, Marigot's abdominal operations. Portal hypertension. McGraw hill companies $12^{\text {th }}$ edition.2013;981-992. 10. Kousei Ishigami, Yan Zhang, Stephen Rayhill, Daniel Katz, and Alan Stolpen. Does Variant Hepatic Artery Anatomy in a Liver Transplant Recipient Increase the Risk of Hepatic Artery Complications After Transplantation?. American Journal of Roentgenology 2004 183:6, 15771584.

11. Erbay N, Raptopoulos V, Pomfret EA, Kamel IR, Kruskal JB. Living Donor Liver Transplantation in Adults: Vascular Variants Important in Surgical Planning for Donors and Recipients. Am J Roentgenol. 2003 July ;181(1):109-14.

12. Kishi Y, Imamura H, Sugawara Y, Sano K, Kaneko J, Kokudo N, et al.
Evaluation of donor vasculobiliary anatomic variations in liver graft procurements. Surgery. 2010 Jan 1;147(1):30-39.

13. Macdonald DB, Haider MA, Khalili K, Kim TK, O'Malley M, Greig PD, et al. Relationship between vascular and biliary anatomy in living liver donors. AJR Am J Roentgenol. 2005 Jul;185(1):247-52.

14. Schroeder T, Malagó M, Debatin JF, Testa G, Nadalin S, Broelsch CE, et al. Multidetector computed tomographic cholangiography in the evaluation of potential living liver donors. Transplantation. 2002 Jun 27;73(12):1972-3.

15. Kishi Y, Sugawara Y, Kaneko J, Akamatsu N, Imamura H, Asato H, et al. Hepatic arterial anatomy for right liver procurement from living donors. Liver Transpl. 2004 Jan 1;10(1):129-133.

16. Takeishi K, Shirabe K, Yoshida Y, Tsutsui Y, Kurihara T, Kimura K, et al.Correlation between Portal Vein Anatomy and Bile Duct Variation in 407 Living Liver Donors. Am J Transplant. 2015 Jan 1;15(1):155-8.

17. Sureka B, Patidar Y, Bansal K, Rajesh S, Agrawal N, Arora A. Portal vein variations in 1000 patients: surgical and radiological importance. Br J Radiol.2015;88(1055):20150326.

18. Covey AM, Brody LA, Getrajdman GI, Sofocleous CT, Brown KT. Incidence, Patterns, and Clinical Relevance of Variant Portal Vein Anatomy. Am J Roentgenol. 2004;Oct 1;183(4):1055-64.

19. Abdoulaye Kanté et al. Morphometry of the Portal Vein: Ultrasound Anatomy about 1000 Cases. Forensic Medicine and Anatomy Research.2019; 07(02):37-43.

20. Kaur $\mathrm{H}$, Singh $\mathrm{M}$, Bajaj AS. A cadaveric study of morphology of portal vein with its clinical importance. Medical Journal of Dr D Y Patil Vidyapeeth. 2016; 9(3): 336-340.

21. Arora J, Kapur V, Kakkar A, Dixit PC. Ramification of portal vein in right lobe of liver.- A corrosion cast study. J Anat Soc India.2003;1:12-14.
Received: Abril 19, 2021

Accepted: May 27, 2021
Corresponding author

Vidya C S

E-mail:vidyacs@jssuni.edu.in 